\title{
A Note on Error Bounds for Convex and Nonconvex Programs*
}

DIMITRI P. BERTSEKAS

bertsekas@LIDS.MIT.EDU

Department of Electrical Engineering and Computer Science, M.I.T., Cambridge, MA, 02139

Received August 7, 1997; Accepted February 19, 1998

\begin{abstract}
Given a single feasible solution $x_{F}$ and a single infeasible solution $x_{I}$ of a mathematical program, we provide an upper bound to the optimal dual value. We assume that $x_{F}$ satisfies a weakened form of the Slater condition. We apply the bound to convex programs and we discuss its relation to Hoffman-like bounds. As a special case, we recover a bound due to Mangasarian [11] on the distance of a point to a convex set specified by inequalities.
\end{abstract}

Keywords: convex programming, optimization, duality, error bounds

Dedication: It is very appropriate that this paper appears in a special issue honoring Olvi Mangasarian, because its idea was actually born while listening to one of Olvi's talks. This is only the latest in a string of several occasions where Olvi's research has substantially influenced mine. For many years, I have benefited a great deal from reading his works, and from interacting with him at meetings, where his presence made serious and idle talk both personally enjoyable and professionally rewarding.

\section{Introduction}

We consider the problem

$$
\begin{array}{ll}
\text { minimize } & f(x) \\
\text { subject to } & x \in X, \quad g_{j}(x) \leq 0, \quad j=1, \ldots, r,
\end{array}
$$

where $X$ is a nonempty subset of $\Re^{n}$, and $f: \Re^{n} \mapsto \Re, g_{j}: \Re^{n} \mapsto \Re$ are given functions. We denote by $g(x)$ the vector of constraint functions

$$
g(x)=\left(g_{1}(x), \ldots, g_{r}(x)\right),
$$

and we write the constraints $g_{j}(x) \leq 0$ compactly as $g(x) \leq 0$. In our notation, all vectors are column vectors and a prime denotes transposition.

${ }^{*}$ Research supported by NSF under Grant 9300494-DMI. 
Let $f^{*}$ and $q^{*}$ be the optimal primal and dual value, respectively:

$$
\begin{aligned}
f^{*} & =\inf _{\substack{x \in X \\
g_{j}(x) \leq 0, j=1, \ldots, r}} f(x), \\
q^{*} & =\sup _{\mu \geq 0} q(\mu),
\end{aligned}
$$

where $q: \Re^{r} \mapsto[-\infty,+\infty)$ is the dual function given by

$$
q(\mu)=\inf _{x \in X}\left\{f(x)+\mu^{\prime} g(x)\right\} .
$$

Throughout the paper, we assume the following:

Assumption 1. We have two vectors $x_{F}$ and $x_{I}$ from $X$ such that:

(a) $x_{F}$ is feasible, i.e., $g\left(x_{F}\right) \leq 0$.

(b) $x_{I}$ is infeasible, i.e., $g_{j}\left(x_{I}\right)>0$ for at least one $j$. Furthermore, its cost $f\left(x_{I}\right)$ is strictly smaller than the cost $f\left(x_{F}\right)$ of $x_{F}$.

We note that by weak duality, we have $q^{*} \leq f\left(x_{F}\right)$. We will show that the value $f\left(x_{I}\right)$ can be used to improve this upper bound. In particular, we prove the following result in Section 3:

Proposition 1. Under Assumption 1, there holds

$$
\frac{q^{*}-f\left(x_{I}\right)}{f\left(x_{F}\right)-f\left(x_{I}\right)} \leq \frac{\Gamma}{\Gamma+1},
$$

where

$$
\Gamma=\inf \left\{\gamma \geq 0 \mid g\left(x_{I}\right) \leq-\gamma g\left(x_{F}\right)\right\}
$$

If $\Gamma=\infty$ because there is no $\gamma \geq 0$ such that $g\left(x_{I}\right) \leq-\gamma g\left(x_{F}\right)$, the bound in Eq. (5) reduces to the trivial bound $q^{*} \leq f\left(x_{F}\right)$.

Note that we have $\Gamma<\infty$ if the Slater condition

$$
g_{j}\left(x_{F}\right)<0, \quad \forall j=1, \ldots, r
$$

holds. More generally, we have $\Gamma<\infty$ if and only if the following weakened form of the Slater condition holds:

$$
g_{j}\left(x_{I}\right) \leq 0 \quad \text { for all } j \text { with } g_{j}\left(x_{F}\right)=0 .
$$

If the above condition holds, we have

$$
\Gamma=\max _{\left\{j \mid g_{j}\left(x_{F}\right)<0\right\}} \frac{g_{j}\left(x_{I}\right)}{-g_{j}\left(x_{F}\right)} .
$$


Figure 1 illustrates the idea underlying the bound (5), (6). In the case of a single constraint $(r=1)$ the bound reduces to

$$
\frac{q^{*}-f\left(x_{I}\right)}{f\left(x_{F}\right)-f\left(x_{I}\right)} \leq \frac{\hat{f}-f\left(x_{I}\right)}{f\left(x_{F}\right)-f\left(x_{I}\right)}=\frac{g\left(x_{I}\right)}{g\left(x_{I}\right)-g\left(x_{F}\right)},
$$

where $\hat{f}$ is the point of intersection of the vertical axis of $\Re^{2}$ with the line segment connecting the vectors $\left(g\left(x_{F}\right), f\left(x_{F}\right)\right)$ and $\left(g\left(x_{I}\right), f\left(x_{I}\right)\right)$. When there are multiple constraints, this line segment can be projected on the two-dimensional subspace spanned by the vertical axis $(0,1)$ of $\Re^{r+1}$ and the vector $\left(g\left(x_{I}\right), 0\right)$. The inequality (10) can then be applied on this subspace in a suitably modified form (see the proof in Section 3 ).

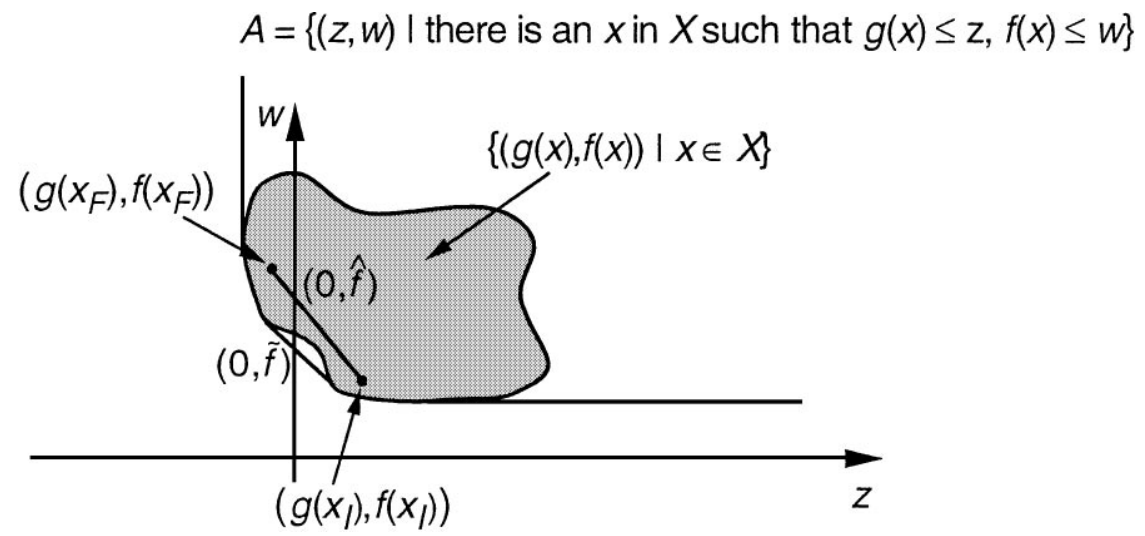

Figure 1. Geometrical interpretation of the bound (8) in the case where there is only one constraint. We consider the convex hull of the subset $A$ of $\Re^{2}$ given by

$$
A=\{(z, w) \mid \text { there exists } x \in X \text { such that } g(x) \leq z, f(x) \leq w\} .
$$

Let $\hat{f}$ be the point of intersection of the vertical axis of $\mathfrak{R}^{2}$ with the line segment connecting the vectors $\left(g\left(x_{F}\right), f\left(x_{F}\right)\right)$ and $\left(g\left(x_{I}\right), f\left(x_{I}\right)\right)$. The vector $(0, \hat{f})$ belongs to $\operatorname{Conv}(A)$. Also, by Euclidean geometry, we have

$$
\frac{\hat{f}-f\left(x_{I}\right)}{f\left(x_{F}\right)-f\left(x_{I}\right)}=\frac{g\left(x_{I}\right)}{g\left(x_{I}\right)-g\left(x_{F}\right)},
$$

and by the definition of $q^{*}$ we have

$$
q^{*} \leq \tilde{f} \leq \hat{f} \leq f^{*},
$$

where

$$
\tilde{f}=\inf \{w \mid(z, w) \in \operatorname{Conv}(A)\} .
$$

Combining these two relations, the bound (5), (6) follows for the case of a single constraint. 
Figure 1 also suggests the following slightly stronger version of our bound:

$$
\frac{\tilde{f}-f\left(x_{I}\right)}{f\left(x_{F}\right)-f\left(x_{I}\right)} \leq \frac{\Gamma}{\Gamma+1},
$$

where

$$
\tilde{f}=\inf \{w \mid(z, w) \in \operatorname{Conv}(A)\}
$$

the subset $A$ of $\Re^{2}$ is given by

$$
A=\{(z, w) \mid \text { there exists } x \in X \text { such that } g(x) \leq z, f(x) \leq w\},
$$

and $\operatorname{Conv}(A)$ denotes its convex hull. Indeed, we prove this bound in Section 3, and we also prove that

$$
\tilde{f} \leq f^{*} .
$$

Furthermore, in the case where $X$ is convex, and $f$ and $g_{j}$ are convex over $X$, we have $\tilde{f}=f^{*}$. We state the corresponding bound as a proposition:

Proposition 2. In addition to Assumption 1, assume that $X$ is convex, and $f$ and $g_{j}$ are convex over $X$. Then, there holds

$$
\frac{f^{*}-f\left(x_{I}\right)}{f\left(x_{F}\right)-f\left(x_{I}\right)} \leq \frac{\Gamma}{\Gamma+1} .
$$

\section{Relations to existing bounds}

There are several analytical and algorithmic contexts where both feasible and infeasible solutions are known in optimization problems (e.g., in primal-dual algorithms), and in which our bound may prove useful. As an illustration of one such context, let us derive an error bound for the Euclidean distance of a point to a convex set specified by inequality constraints. A similar error bound for this projection problem was derived by Mangasarian [11] using different methods, and was the inspiration for the present paper. In particular, let $y \in \Re^{n}$ be a given vector and consider the following projection problem

$$
\begin{array}{ll}
\operatorname{minimize} & \|y-x\| \\
\text { subject to } & x \in X, \quad g_{j}(x) \leq 0, \quad j=1, \ldots, r,
\end{array}
$$

where $\|\cdot\|$ denotes the standard Euclidean norm $\left(\|x\|=\sqrt{\sum_{i=1}^{n} x_{i}^{2}}\right)$. Let us assume that $X$ is a convex set and $g_{j}$ are convex functions. Furthermore, let us assume the following two conditions: 
(a) $y \in X$ and $g_{j}(y)>0$ for at least one $j$.

(b) There exists a vector $x_{F} \in X$ such that

$$
\begin{aligned}
g_{j}\left(x_{F}\right) \leq 0, \quad \forall j=1, \ldots, r, \\
g_{j}(y) \leq 0 \quad \text { for all } j \text { with } g_{j}\left(x_{F}\right)=0 .
\end{aligned}
$$

Then we can apply the error bound (13) with $f(x)=\|y-x\|, f^{*}$ equal to the distance $d(y)$ of $y$ from the convex set $X \cap\left\{x \mid g_{j}(x) \leq 0, j=1, \ldots, r\right\}, x_{I}=y$, and $f\left(x_{I}\right)=0$, $f\left(x_{F}\right)=\left\|y-x_{F}\right\|$. We have

$$
d(y) \leq \frac{\Gamma}{\Gamma+1}\left\|y-x_{F}\right\|,
$$

where $\Gamma$ is given by Eq. (9). It is easily seen that $\Gamma \leq \hat{\Gamma}$, where

$$
\hat{\Gamma}=\frac{\max _{\left\{j \mid g_{j}\left(x_{F}\right)<0\right\}} g_{j}\left(x_{I}\right)}{\min _{\left\{j \mid g_{j}\left(x_{F}\right)<0\right\}}-g_{j}\left(x_{F}\right)},
$$

and the inequality (14) yields

$$
d(y) \leq \frac{\hat{\Gamma}}{\hat{\Gamma}+1}\left\|y-x_{F}\right\|,
$$

or equivalently

$$
d(y) \leq \frac{\max _{\left\{j \mid g_{j}\left(x_{F}\right)<0\right\}} g_{j}\left(x_{I}\right)}{\max _{\left\{j \mid g_{j}\left(x_{F}\right)<0\right\}} g_{j}\left(x_{I}\right)+\min _{\left\{j \mid g_{j}\left(x_{F}\right)<0\right\}}-g_{j}\left(x_{F}\right)}\left\|y-x_{F}\right\| .
$$

This bound coincides with the relative error bound derived by Mangasarian ([11], Theorem 2.2) under the assumption that $X=\Re^{n}$ and $g_{j}\left(x_{F}\right)<0$ for all $j$. Note, however, that using $\Gamma$ from Eq. (9) in place of $\hat{\Gamma}$ as in Eq. (16) yields a stronger bound.

For a generalization of the bound (14), let us consider replacing the distance $\|y-x\|$ with a more general metric. In particular, consider the problem

$$
\begin{array}{ll}
\operatorname{minimize} & f(x, y) \\
\text { subject to } & x \in X, \quad g_{j}(x) \leq 0, \quad j=1, \ldots, r,
\end{array}
$$

where $X$ is a convex set, $g_{j}$ is a convex function, and $f(\cdot, y)$ is a convex function satisfying

$$
f(y, y)=0, \quad f\left(x_{F}, y\right)>0 .
$$

Then, if $f^{*}(y)$ is the optimal cost of this problem, the preceding analysis can be used to show that (cf. Eq. (14))

$$
f^{*}(y) \leq \frac{\Gamma}{\Gamma+1} f\left(x_{F}, y\right),
$$


where $\Gamma$ is given by

$$
\Gamma=\max _{\left\{j \mid g_{j}\left(x_{F}\right)<0\right\}} \frac{g_{j}(y)}{-g_{j}\left(x_{F}\right)} .
$$

In Mangasarian's paper [11], the bound (17) was used to derive an extension to Hoffman's error bound for the approximate solution of convex systems of inequalities. Hoffman's bound [4] and its extensions have been the subject of intensive investigation recently (see e.g., [2, 5-12], which give many additional references). It is thus interesting to inquire about the relation of our bound of Section 1 with Hoffman-like bounds. It turns out that the bound of Section 1 is fundamentally different. To see this, we note that Propositions 1 and 2 do not explicitly require the existence of a Lagrange multiplier for problem (1). By contrast, we will show shortly that Hoffman-like bounds essentially amount to assertions on the uniform boundedness of the Lagrange multipliers of some parametric convex program as the parameter vector ranges over some set. This connection, which is based on the theory of exact penalty functions, has apparently not been made explicit earlier and leads to slightly stronger versions of Hoffman's bound for convex systems of inequalities than some of those available in the literature (e.g., Mangasarian's Hoffman-like bound of [11]).

Indeed let $Y$ be a subset of $\Re^{n}$, let $y$ be a parameter vector taking values in $Y$, and consider the parametric program

$$
\begin{array}{ll}
\operatorname{minimize} & f(x, y) \\
\text { subject to } & x \in X, \quad g_{j}(x, y) \leq 0, \quad j=1, \ldots, r,
\end{array}
$$

where $X$ is a convex subset of $\Re^{n}$, and for each $y \in Y, f(\cdot, y)$ and $g_{j}(\cdot, y)$ are convex functions. We assume that for each $y \in Y$, this program has at least one feasible solution, and that its optimal value, denoted $f^{*}(y)$, is finite. Furthermore, we assume that when the constraints $g_{j}(x, y) \leq 0$ are dualized, there is no duality gap; that is, the optimal value $q^{*}(y)$ of the dual problem

$$
\begin{array}{ll}
\text { maximize } & q(\mu, y) \\
\text { subject to } & \mu \geq 0
\end{array}
$$

is equal to $f^{*}(y)$, where $q(\mu, y)$ is the dual function

$$
q(\mu, y)=\inf _{x \in X}\left\{f(x, y)+\mu^{\prime} g(x, y)\right\} .
$$

Consider a penalty function $P: \Re^{r} \mapsto \Re$ that is convex and satisfies

$$
\begin{array}{ll}
P(u)=0, & \forall u \leq 0, \\
P(u)>0, & \text { if } u_{j}>0 \text { for some } j=1, \ldots, r .
\end{array}
$$

Let $c>0$ denote a penalty parameter. It is shown in [1] [Proposition 5.4.1(a) $]^{1}$ that we have

$$
f^{*}(y)=\inf _{x \in X}\{f(x, y)+c P(g(x, y))\}, \quad \forall y \in X,
$$


if and only if

$$
u^{\prime} \mu^{*}(y) \leq c P(u), \quad \forall u \in \Re^{r}, \forall y \in X,
$$

for some dual optimal solution $\mu^{*}(y)$ (an optimal solution of the dual problem (14), which is also referred to as a Lagrange multiplier).

It is seen that Eq. (19) is equivalent to the bound

$$
f^{*}(y) \leq f(x, y)+\bar{c} P(g(x, y)), \quad \forall x \in X, y \in Y,
$$

so this bound holds if and only if there exists a uniform bounding constant $c>0$ such that

$$
u^{\prime} \mu^{*}(y) \leq c P(u), \quad \forall u \in \Re^{r}, y \in Y
$$

For the above relation to hold, it is essential that the penalty function $P$ be nondifferentiable, such as for example the Euclidean norm of the vector $u^{+}$that has components $\max \left\{0, u_{j}\right\}$, $j=1, \ldots, r$,

$$
P(u)=\left\|u^{+}\right\|,
$$

or the corresponding $l_{1}$ or $l_{\infty}$ norm

$$
P(u)=\left\|u^{+}\right\|_{1}, \quad P(u)=\left\|u^{+}\right\|_{\infty} .
$$

Given any of these choices, it is seen that Eq. (21), and the equivalent bound (20), hold if and only if for every $y \in Y$, it is possible to select a Lagrange multiplier $\mu^{*}(y)$ of the parametric problem (18) such that the set $\left\{\mu^{*}(y) \mid y \in Y\right\}$ is bounded.

Let us now specialize the preceding discussion to the parametric program

$$
\begin{array}{ll}
\operatorname{minimize} & f(x, y)=\|y-x\| \\
\text { subject to } & x \in X, \quad g_{j}(x) \leq 0, \quad j=1, \ldots, r,
\end{array}
$$

where $\|\cdot\|$ is the Euclidean norm, $X$ is a convex subset of $\Re^{n}$, and $g_{j}$ are convex functions. This is the projection problem considered in the beginning of this section. Let us take $Y=X$. If $c$ satisfies Eq. (21), the bound (20) becomes

$$
d(y) \leq\|y-x\|+c P(g(x)), \quad \forall x \in X, y \in X,
$$

and (by taking $x=y$ ) implies the bound

$$
d(y) \leq c P(g(y)), \quad \forall y \in X .
$$

Thus, the Hoffman-like bound (23) holds if a Lagrange multiplier $\mu^{*}(y)$ of the projection problem (22) can be found such that Eq. (21) holds. In the case where $P$ is given by

$$
P(u)=\left\|u^{+}\right\|,
$$


(or $P$ is given by any one of a variety of other nondifferentiable functions of $u$ ) boundedness of the set $\left\{\mu^{*}(y) \mid y \in X\right\}$ is equivalent to the condition (21), and implies the Hoffman-like bound (23). It turns out that the reverse assertion also holds, as shown in the following proposition:

Proposition 3. Let $d(y)$ be the optimal value of the projection problem

$$
\begin{array}{ll}
\text { minimize } & \|y-x\| \\
\text { subject to } & x \in X, \quad g_{j}(x) \leq 0, \quad j=1, \ldots, r,
\end{array}
$$

$X$ is a convex subset of $\Re^{n}$, and the functions $g_{j}$ are convex. The Hoffman-like bound

$$
d(y) \leq c\left\|(g(y))^{+}\right\|, \quad \forall y \in X,
$$

holds for some constant $c$ if and only if the projection problem (24) has a Lagrange multiplier $\mu^{*}(y)$ such that the set $\left\{\mu^{*}(y) \mid y \in X\right\}$ is bounded.

Proof: The preceding discussion showed that the boundedness condition on the Lagrange multipliers implies the Hoffman-like bound (25), so there remains to prove the reverse assertion. Indeed, assume that Eq. (25) holds for some $c$, and to arrive at a contradiction, assume that there exist $x \in X$ and $y \in Y$ such that

$$
d(y)>\|y-x\|+c\left\|(g(x))^{+}\right\| .
$$

Then, using Eq. (25), we obtain

$$
d(y)>\|y-x\|+d(x)
$$

From this relation and the triangle inequality, it follows that

$$
\begin{aligned}
\inf _{z \in X, g(z) \leq 0}\|y-z\| & >\|y-x\|+\inf _{z \in X, g(z) \leq 0}\|x-z\| \\
& =\inf _{z \in X, g(z) \leq 0}\{\|y-x\|+\|x-z\|\} \\
& \geq \inf _{z \in X, g(z) \leq 0}\|y-z\|,
\end{aligned}
$$

which is a contradiction. Thus, Eq. (25) implies that we have

$$
d(y) \leq\|y-x\|+c\left\|(g(x))^{+}\right\|, \quad x \in X, y \in X .
$$

Using Proposition 5.4.1(a) of [1], this implies that there exists a Lagrange multiplier $\mu^{*}(y)$ such that

$$
u^{\prime} \mu^{*}(y) \leq c\left\|u^{+}\right\|, \quad \forall u \in \Re^{r}, y \in X
$$


This in turn implies the boundedness of the set $\left\{\mu^{*}(y) \mid y \in X\right\}$.

Let us give two conditions under which the boundedness condition of Proposition 3 is satisfied and a Hoffman-like bound of the form (25) holds:

(a) $X=\Re^{n}$ and $g_{j}$ are linear (this is the original Hoffman's bound [4]). For a simple way to prove this, let $g_{j}(x)=a_{j}^{\prime} x-b_{j}$, where $a_{j}$ is a vector in $\Re^{n}$ and $b_{j}$ is a scalar. Then, because of the linearity of the constraints, the projection problem (24) has at least one Lagrange multiplier $\mu^{*}(y)$ (see e.g., [1], p. 437). This Lagrange multiplier satisfies

$$
\frac{y-\hat{y}}{\|y-\hat{y}\|}=\sum_{j \in J(y)} \mu_{j}^{*}(y) a_{j}
$$

where $\hat{y}$ is the unique projection of $y$, and $J(y)$ is a subset of indices such that the set of vectors $\left\{a_{j} \mid j \in J(y)\right\}$ is linearly independent. (We assume here that $y \neq \hat{y}$, which is the case of interest.) Since the vector $\sum_{j \in J(y)} \mu_{j}^{*}(y) a_{j}$ has norm 1, it follows that $\mu^{*}(y)$ can be selected so that the set $\left\{\mu^{*}(y) \mid y \in \Re^{n}\right\}$ is bounded.

(b) For each $y \in X$, a Slater condition holds; that is there exists a vector $\bar{x}(y) \in X$ such that $g_{j}(\bar{x}(y))<0$ for all $j=1, \ldots, r$. Furthermore, there is a constant $\gamma$ such that

$$
\frac{\|y-\bar{x}(y)\|-d(y)}{\min _{j=1, \ldots, r}\left\{-g_{j}(\bar{x}(y))\right\}} \leq \gamma, \quad \forall y \in X .
$$

Mangasarian [11] used these two conditions (together with the additional condition $X=\Re^{n}$ ) to obtain a Hoffman-like bound of the form (25). For an alternative proof of this result, note that the Slater condition implies (see e.g., [1], p. 450 or [3], p. 313) that for each $y \in X$ there exists a Lagrange multiplier $\mu^{*}(y)$ with

$$
\sum_{j=1}^{r} \mu_{j}^{*}(y) \leq \frac{\|y-\bar{x}(y)\|-d(y)}{\min _{j=1, \ldots, r}\left\{-g_{j}(\bar{x}(y))\right\}} .
$$

Thus, Eq. (26) implies the boundedness condition of Proposition 3.

\section{Proof of Propositions 1 and 2}

We consider the subset of $\mathfrak{R}^{r+1}$

$$
A=\{(z, w) \mid \text { there exists } x \in X \text { such that } g(x) \leq z, f(x) \leq w\},
$$

and its convex hull $\operatorname{Conv}(A)$. The vectors $\left(g\left(x_{F}\right), f\left(x_{F}\right)\right)$ and $\left(g\left(x_{I}\right), f\left(x_{I}\right)\right)$ belong to $A$. In addition, the vector $(0, \tilde{f})$, where

$$
\tilde{f}=\inf \{w \mid(z, w) \in \operatorname{Conv}(A)\},
$$


is in the closure of $\operatorname{Conv}(A)$. Let us now show that $q^{*} \leq \tilde{f}$, as indicated by figure 1 .

Indeed, for each $(z, w) \in \operatorname{Conv}(A)$, there exist $\xi_{1} \geq 0$ and $\xi_{2} \geq 0$ with $\xi_{1}+\xi_{2}=1$, and $x_{1} \in X, x_{2} \in X$ such that

$$
\begin{gathered}
\xi_{1} g\left(x_{1}\right)+\xi_{2} g\left(x_{2}\right) \leq z, \\
\xi_{1} f\left(x_{1}\right)+\xi_{2} f\left(x_{2}\right) \leq w .
\end{gathered}
$$

Furthermore, by the definition of the dual function $q$, we have for all $\mu \in \mathfrak{R}^{r}$,

$$
\begin{aligned}
& q(\mu) \leq f\left(x_{1}\right)+\mu^{\prime} g\left(x_{1}\right), \\
& q(\mu) \leq f\left(x_{2}\right)+\mu^{\prime} g\left(x_{2}\right) .
\end{aligned}
$$

Combining the preceding four inequalities, we obtain

$$
q(\mu) \leq w+\mu^{\prime} z, \quad \forall(z, w) \in \operatorname{Conv}(A), \mu \geq 0 .
$$

The above inequality holds also for all $(z, w)$ that are in the closure of $\operatorname{Conv}(A)$, and in particular, for $(z, w)=(0, \tilde{f})$. It follows that

$$
q(\mu) \leq \tilde{f}, \quad \forall \mu \geq 0,
$$

from which, by taking the maximum over $\mu \geq 0$, we obtain $q^{*} \leq \tilde{f}$.

Let $\gamma$ be any nonnegative scalar such that $g\left(x_{I}\right) \leq-\gamma g\left(x_{F}\right)$, and consider the vector

$$
\Delta=-\gamma g\left(x_{F}\right)-g\left(x_{I}\right) .
$$

Since $\Delta \geq 0$, it follows that the vector

$$
\left(-\gamma g\left(x_{F}\right), f\left(x_{I}\right)\right)=\left(g\left(x_{I}\right)+\Delta, f\left(x_{I}\right)\right)
$$

also belongs to the set $A$. Thus the three vectors

$$
\left(g\left(x_{F}\right), f\left(x_{F}\right)\right), \quad(0, \tilde{f}), \quad\left(-\gamma g\left(x_{F}\right), f\left(x_{I}\right)\right)
$$

belong to the closure of $\operatorname{Conv}(A)$, and form a triangle in the plane spanned by the "vertical" vector $(0,1)$ and the "horizontal" vector $\left(g\left(x_{F}\right), 0\right)$.

Let $(0, \hat{f})$ be the intersection of the vertical axis with the line segment connecting the vectors $\left(g\left(x_{F}\right), f\left(x_{F}\right)\right)$ and $\left(-\gamma g\left(x_{F}\right), f\left(x_{I}\right)\right)$ (there is a point of intersection because $\gamma \geq 0$ ). We have by Euclidean triangle geometry (cf. figure 1)

$$
\frac{\hat{f}-f\left(x_{I}\right)}{f\left(x_{F}\right)-f\left(x_{I}\right)}=\frac{\gamma}{\gamma+1} .
$$

Since the vectors $\left(g\left(x_{F}\right), f\left(x_{F}\right)\right)$ and $\left(-\gamma g\left(x_{F}\right), f\left(x_{I}\right)\right)$ both belong to $\operatorname{Conv}(A)$, we also have $(0, \hat{f}) \in \operatorname{Conv}(A)$. Therefore, there exist vectors $x_{1}, \ldots, x_{m} \in X$ and nonnegative scalars $\xi_{1}, \ldots, \xi_{m}$ with $\sum_{i=1}^{m} \xi_{i}=1$, satisfying

$$
\sum_{i=1}^{m} \xi_{i} g\left(x_{i}\right) \leq 0, \quad \sum_{i=1}^{m} \xi_{i} f\left(x_{i}\right) \leq \hat{f} .
$$


Thus, if $\hat{f}<\tilde{f}$, we must have $\sum_{i=1}^{m} \xi_{i} f\left(x_{i}\right)<\tilde{f}$, contradicting the definition of $\tilde{f}$. It follows that $\tilde{f} \leq \hat{f}$ and since $q^{*} \leq \tilde{f}$, as shown earlier, from Eq. (27) we obtain

$$
\frac{q^{*}-f\left(x_{I}\right)}{f\left(x_{F}\right)-f\left(x_{I}\right)} \leq \frac{\tilde{f}-f\left(x_{I}\right)}{f\left(x_{F}\right)-f\left(x_{I}\right)} \leq \frac{\gamma}{\gamma+1} .
$$

Taking the infimum over $\gamma \geq 0$, the error bound (5), (6) follows.

Assume now that $X$ is convex, and $f$ and $g_{j}$ are convex over $X$. Then the set $A$ is known to be convex under these assumptions (see e.g., [1], Proposition 5.3.1, p. 446), and we have $\tilde{f}=f^{*}$. Proposition 2 then follows from Eq. (28).

\section{Note}

1. Note that Proposition 5.4.1(a) of [1] includes a compactness assumption on the set $X$, but this assumption is unnecessary as long as finiteness of the optimal value $f^{*}(y)$ is assumed; see the discussion of p. 473 of [1].

\section{References}

1. D.P. Bertsekas, Nonlinear Programming, Athena Scientific, Belmont, MA, 1995.

2. J.V. Burke and P. Tseng, "A unified analysis of Hoffman's error bound via Fenchel duality," SIAM J. on Optimization, vol. 6, pp. 265-282, 1996.

3. J.-B. Hiriart-Urruty and C. Lemarechal, "Convex analysis and minimization algorithms," vols. I and II, Springer-Verlag: Berlin and NY, 1993.

4. A.J. Hoffman, "On approximate solutions of systems of linear inequalities," Natl. Bur. Standards, vol. 49, pp. 263-265, 1952

5. D. Klatte and W. Li, "Asymptotic constraint qualifications and global error bounds for convex inequalities," Math. Programming, 1997, to appear.

6. A.S. Lewis and J.-S. Pang, "Error bounds for convex inequality systems," in Proceedings of the Fifth Symposium on Generalized Convexity, Luminy, J.P. Crouzeix, J.-E. Martinez-Legaz, and M. Volle (Eds.), June 1996, Kluwer Academic Publishers, 1998, pp. 75-110.

7. W. Li, "Abadie's constraint qualification, metric regularity, and error bounds for differentiable convex inequalities," SIAM J. on Optimization, vol. 7, pp. 966-978, 1997.

8. X.-D. Luo and Z.-Q. Luo, "Extension of Hoffman's error bound to polynomial systems," SIAM J. on Optimization, vol. 4, pp. 383-392, 1994.

9. Z.-Q. Luo and P. Tseng, "Error bound and convergence analysis of matrix splitting algorithms for the affine variational inequality problem," SIAM J. on Optimization, vol. 2, pp. 43-54, 1992.

10. Z.-Q. Luo and P. Tseng, 1992. "On the linear convergence of convex essentially smooth minimization," SIAM J. on Control and Optimization, vol. 30, pp. 408-425, 1992.

11. O.L. Mangasarian, "Error bounds for nondifferentiable convex inequalities under a strong Slater constraint qualification,” Mathematical Programming, vol. 83, pp. 187-194, 1998.

12. J.-S. Pang, "Error bounds in mathematical programming," Math. Programming, vol. 79, pp. 299-332, 1997. 